\title{
Trends in Education-specific Differences in Disability-Free Life Expectancy in Spain, 2008-2017
}

\author{
Mathias Voigt \\ Institute of Economy Geography and Demography \\ CSIC-CCHS \\ Calle Albasanz 24-26 \\ 28037 Madrid \\ Tel.: +34657682637 \\ mathias.voigt@cchs.csic.es \\ Sebastian Daza \\ Institute of Economy, Geography and Demography \\ CSIC-CCHS \\ Dariya Ordanovich \\ Institute of Economy, Geography and Demography \\ CSIC-CCHS \\ Alberto Palloni \\ Institute of Economy, Geography and Demography \\ CSIC-CCHS
}

September 8, 2020

\footnotetext{
*Working paper. Please, do not cite without permission. This project has received funding from the European Research Council (ERC) under the European Union's Horizon 2020 research and innovation programme (grant agreement No 788582). This publication reflects only the author(s)'s view and the Research Executive Agency and the Commission are not responsible for any use that may be made of the information it contains.
} 


\section{ABSTRACT}

Background: There is mounting evidence for a recent increase of social disparities in chronic disease prevalence and mortality. However, little is known about how these trends are reflected in combined measures of morbidity, disability and mortality.

Method: We use two nationally representative surveys of the Spanish population for the years 2008 to 2017 and standard measures of expected duration of disability and illness to assess time

trends and social disparities in mortality, morbidity and expected years lived in disability (DFLE) and with chronic illness (chrDFLE). We provide empirical evidence of shifting trends for these measures. We then decompose these changes into contributions associated with disability, chronic illness and mortality. Finally, we estimate the size of education differentials in DFLE and chrDFLE and evaluate the magnitude and direction of changes of these differentials over time.

Results: While the disability based indicator suggests a decrease of expected years without disability for both men and women (expansion of morbidity), the morbidity based indicator shows an increase in time spend free of chronic disease for women but a slight decrease for men. The decrease in time spent without disability was observed for all education groups but is particularly marked for those with low education.

Conclusion: We find evidence of an expansion of morbidity in Spain between 2008 and 2017. The bulk of this development is related to increases in time spent with functional limitations over this period. These patterns occur in conjuncture with growing social disparities in time spend with chronic illness or disability.

Key Words: Compression of Morbidity, Disability-free Life Expectancy, Chronic Disease, Educational Disparities 


\section{Introduction}

Jointly with reductions of fertility, the continuous mortality decline at ages 65 and older have transformed the age compositions of the populations with the net result that a higher fraction of the population survives to older ages and simultaneously, is expected to live longer lives [1--3]. A key question is whether the extra years lived by the growing population of elderly are spent in good health or in illness or disability. As empirical evidence of increasing social disparities in morbidity, disability and mortality is growing [4, 5], it becomes important to assess if additional or lost years with good health are shared equally across different social groups.

The Spanish case is an example of the joint occurrence of steady, if unequal, improvements in mortality and stalling or increasing deterioration of the average duration of life lived in good health. Fueled by decreasing trends of cardiovascular diseases (CVD) and hypertension, Spain has experienced a rapid reduction of mortality rates over the past 50 years or so [3]. Although there has been no sign of slowing mortality improvements, it can be assumed that further declines in these conditions are unlikely to continue sustaining additional improvements in survival.

It should not, then, be a surprise to find that past studies combining Spanish mortality and morbidity trends suggest a shift from a regime with improvements in expected life lived healthy to a more recent one dominated by deterioration. Indeed, while empirical findings suggested compression of morbidity in the 1980 and 1990s [6], more recent analyses point toward an expansion of morbidity dating back since the beginning of the $21^{\text {st }}$ century [7, 8].

If verified, these patterns suggest a shifting landscape that will alter Spain's profile of mortality, morbidity and disability. In what follows, we review evidence supporting these empirical findings and expand them in three ways. First, we examine two indicators of healthy life expectancy, one associated with disability and the other with chronic diseases. Second, we decompose changes to identify the most important culprits for the deterioration of these indicators. Third, and finally, we

discuss time trends of educational differentials caused by both shifts in differential mortality and prevalence of chronic conditions. 


\section{Background}

There are three competing theories that offer contrasting predictions of future population health scenarios. The "failure of success" perspective invokes a rather bleak scenario as it predicts that improvements in survival at older adult ages will be accompanied by increased disease prevalence due to improved survival of individuals with chronic illnesses [9].

The original compression of morbidity hypothesis [10] argues that the average age of onset of chronic disease will be shifted towards older ages at a faster pace than improvements in survival, thus leading to shorter periods of morbidity and a concentration of illnesses at very old ages (i.e. producing "compression of morbidity").

Finally, a third conjecture by Manton [11] proposes that, as a consequence of multiple advances, principally medical innovations, most diseases will be controlled at early stages of their progression thus reducing the average severity of chronic conditions and lead to a "dynamic equilibrium" with no systematic compression or expansion of morbidity [11, 12].

Empirical evidence gathered in the last twenty or so years supporting or disproving the three theories is mixed. There are studies confirming the existence of compression in countries with exceptionally low mortality and only when severe, rather than all, limitations are accounted for [13, 14]. Other research demonstrates the opposite, namely, it shows that elderly populations in many countries spend more time in disability and with chronic diseases when compared with older generations, suggesting an expansion of morbidity [8, 15, 16]. Furthermore, a number of studies find a combination of trends such as a decline of time spent with functional limitations occurring simultaneously with an increase in the duration of chronic diseases. Finally, there is also evidence for shifting landscapes over time in the same population, the result of a replacement of conditions that produce compression by those sustaining expansion or vice-versa [12].

The Spanish case is a good example of this replacement scenario, as new studies point to a shift from a period characterized by morbidity compression towards a recent expansion [7, 8].

What factors could account for this transition from a regime characterized by compression of morbidity and disability to one with signs of expansion? Although the pace of Spanish mortal- 
ity decline has slowed down, it continues to occur, albeit with growing disparities across social classes. Under these conditions, expansion of morbidity and disability can only occur if there is either increased duration and/or increased prevalence of chronic illnesses and disability. This scenario is quite likely to be occurring. In fact, the period during which there was increased control of CVD diseases and that led to important morbidity and mortality improvements after 1970 or so is reaching an exhaustion point. Simultaneously, Spain, like other middle and high income countries, is experiencing increased prevalence of obesity, Type II Diabetes (T2D) and related comorbidities, life style associated cancers (lung and stomach cancer) and neuro-degenerative diseases [17--19]. These chronic conditions and illnesses are important contributors to increases in duration spent in unhealthy states. Furthermore, because these conditions strike different social strata unequally, they might also be an important cause of disparities in adult life expectancy and healthy life expectancy [20, 21].

This suggests that the Spanish current patterns of morbidity and mortality should be characterized by two new features. The first is the presence of signs of expansion of morbidity and disability for which, if verified empirically, the question to answer is what conditions (chronic illnesses, disabilities, mortality) account for this trend?

The second new feature is the potential increase in social disparities. In the past twenty years or so, a large body of research has documented persistent social disparities in morbidity, disability and mortality in Spain and other European countries [4, 5, 22]. If these were sustained under conditions of morbidity and disability compression, could a shift to a regime characterized by expansion lead to a widening of healthy life expectancy inequalities? It is not immediately obvious, since there are multiple possible combinations of mortality, morbidity and disability patterns, different compositions of disparities in them may generate conditions for either contraction or expansion of healthy life expectancy inequalities. If the empirical evidence points toward increases rather than decreases in disparities, what are their most proximate determinants [23-25]? 


\section{Methods}

We use two data sets. The first is the National Survey on Disability, Personal Autonomy and Dependency (EDAD), a nationally representative survey study conducted by the Spanish National Institute of Statistics (INE) in 2008. This data set is linked to death records for the period between 2008 to 2017 that we use to estimate mortality rates and compute life tables in five year age groups from age 65 to $90+$ by sex and education. The estimated life tables apply to two periods, one centered on the interval 2008-2012 and the other centered on the interval 2013-2017 1 We also use the EDAD survey to estimate prevalence rates of disability and selected chronic conditions by age, sex and education for the period 2008-2012. These rates and the life table associated with the first period are then used to estimate expected years of disability free life expectancy at age 65 $\left(D_{F L E}\right)$ and years of life expectancy free of chronic disease $\left(\text { chrDFLE }_{65}\right)^{2}$.

For the second period (2013-2017), we compute prevalence rates of disability and chronic illness by age, sex and education using information from the Spanish National Health Survey 2017 (ENSE), which is also conducted by INE. These rates are then combined with the life table for 2013-2017 to estimate the two indicators of healthy life expectancy 3 . For both years, we group individuals according to their highest obtained education and collapse them into three categories: no completed formal education (1), completed primary education (2), and secondary or higher education (3).

\subsection{Disability}

Disability is defined as the occurrence of a functional limitation in at least one of five basic activities of daily living (ADL), which include Eating and Drinking, Transferring, Getting dressed, Toiletting, Basic Hygiene. Age-specific fractions of people with disability are estimated by sex

\footnotetext{
${ }^{1}$ The INE Department for Socio-Demographic Statistics linked 207,529 of a total 258,187 individuals between the ages 0 and 104 to administrative mortality and exposure data for the period between 2008 and 2017 . Survey participants were linked to the annually updated statistics of natural population movements (MNP) and the yearly updated population register (Spanish: Padrón) via an individual identifier

${ }^{2}$ The technical details of the construction of the two indicators are in the Appendix.

${ }^{3}$ see Appendix
} 
and education for the two different points in time. Fractions for the first period are estimated from age-specific prevalence observed in EDAD. They are then combined with the life tables for this period using Sullivan's method to construct the index of expected years of disability free life expectancy [26]. Standard errors and 95\% confidence intervals are computed via bootstrap. We then use an extension of Arriaga's decomposition technique [27] for changes in life expectancy applied to DFLE to decompose DFLE time trends for both sexes and all education groups into additive contributions due to changes in mortality rates and disability [28].

\subsection{Chronic Conditions}

The assessment of morbidity based on functional limitations solely can be misleading since these indicators are partly influenced by changes in the environments to which individuals are exposed and can vary by personal preferences, conceptions and perceptions about capabilities and limitations [29]. To circumvent this, we propose examination of an indicator of life expectancy free of diagnosed chronic conditions. We select four groups of diseases that are most frequent in modern, older populations: Type II Diabetes (T2D), heart disease, cerebrovascular diseases/incidents, and cancer. We then estimate the age, sex and education-specific prevalence of individuals with at least one of these conditions and combine them with the life tables for the second period to obtain expected years lived without chronic disease at age $65\left(\operatorname{chrDFLE}_{65}\right)$. As before, we estimate standard errors and $95 \%$ confidence intervals via bootstrap and decompose changes in chrDFLE 65 over time and between education groups.

\section{Results}

\subsection{Disability Patterns and Trends}

The fraction of individuals with functional limitations increases with age for all groups and suggests that the majority of both women older than 95 and men age 90 and older experience some 
form of limitations in at least one of the ADLs. The magnitude of these rates are somewhat higher in 2017 for both genders. As shown in Fig. 1, the proportions of women with disability are higher when compared with men in the same educational group. During both periods, highly educated men exhibit the lowest levels of functional limitations. Differences between the two highest education groups are modest but are much sharper when the contrast is with the lowest educated group.

\subsection{Chronic Disease Patterns and Trends}

By and large, chronic disease prevalence rates are higher than prevalence of disability rates. This confirms that inferences about morbidity should not be solely based on disability but complemented by an indicator based on prevalence of chronic illnesses 4 . Education-specific prevalence of chronic diseases are displayed in Fig. S1. Asides from the fact that figures are larger in the second period and involve a slight disadvantage for men, there are no systematic patterns.

\subsection{Mortality Patterns and Trends}

As described before, mortality rates are estimated from the linked EDAD follow-up study and used to construct life tables starting at age 65 for the periods 2008-2012 and 2013-2017. When compared with official vital statistics, our life expectancy estimates underestimate somewhat total mortality at younger ages and overestimate it at ages over 80 . The differences, however, are very small.

Table $\mathrm{S} 1$ in the appendix displays life expectancy estimated by education and gender with $95 \%$ confidence intervals. It shows marginal increases in life expectancy at most ages for the lowest and highest education group and for both genders. While there is a clear relationship between education and mortality, it is much more pronounced at younger ages. Female educational disparities prevail at all ages whereas males' differentials attenuate and vanish at very old ages.

\footnotetext{
${ }^{4}$ The downside of the chronic disease-based indicator is that it depends on a complex convolution of multiple ages of onset (diagnosis) of each of the chronic illnesses selected and, therefore, on idiosyncrasies of screening and diagnosis characteristic of each of them.
} 


\subsection{Disability-Free and Chronic Disease-Free Life Expectancy}

\subsubsection{Disability-Free Life Expectancy}

As conjectured before, trends of DFLE $_{65}$ of disability-free life expectancy show reductions for both men and women between the two periods (in Appendix Table S2). Women exhibit higher DFLE $_{65}$ than men and also experienced larger declines $(-0.74$ years, 95\% CI $[-0.96,-0.52])$ when compared to men $(-0.36$ years, $95 \%$ CI $[-0.61,-0.11])$.

Education-specific changes in DFLE $_{65}$ are displayed in Table 1. In general, it is those with the lowest levels of education that experience the lowest levels of DFLE. The other two education groups exhibit very similar levels. Note also that DFLE $_{65}$ has decreased between the two periods for all education groups and both genders and that magnitude of decline is larger for women and men with lowest education. Among men it is only those with lowest education that experience a substantial reduction in $\operatorname{DFLE}_{65}$ of more than a year $(1.33$ years , 95\% CI $\left.[1.26,1.86])\right)$. The remaining education groups undergo only small decreases. Among women, for both those with low and with high education, there is evidence of a reduction of $\mathrm{DFLE}_{65}$ by more than a year between the two periods

\subsubsection{Chronic disease-free life expectancy}

In contrast to the $\mathrm{DFLE}_{65}$ trends, estimates of chronic disease free life expectancy are more mixed. On one hand, among men there is a systematic decrease in chrDFLE in all educational groups. Females, on the other hand, experienced increases in time spend without chronic disease. This pattern is more pronounced among those with primary or higher education and induces increases in education differentials in chrDFLE 65 at least among females.

\subsection{Decomposition}

The results from our decomposition exercise, shown in Table 2, reveal that the decline of DFLE is attributable almost exclusively to additional live years spend with disability (Disability Effect 
- DE) rather than to changes in mortality (Mortality Effect - ME), a pattern most marked among women with low education (i.e. $\Delta D E_{65}=-1.59$ years, $95 \%$ CI $[-2.19,-0.92]$ ) 5 and secondary or higher education (i.e. $\Delta D E_{65}=-1.39$ years, $95 \%$ CI $[-2.91,-0.49]$ ). On the other hand, men with low education experienced the greatest decline in $D F L E_{65}\left(\triangle D F L E_{65}-1.33\right.$ years , 95\% CI $[-1.96,-0.61])$, and is mostly attributable to increased levels of disability in all age groups $\left(\Delta D E_{65}=-1.07\right.$ years, $\left.95 \% \mathrm{CI}[-1.61,-0.48]\right)$.

Differences overtime between the lowest and two highest education groups (see Appendix Table $[\mathrm{S} 3 \mathrm{p}$ increase mostly as a result of disparities of mortality and disability trends among women. In turn, these differences are driven by increases in disability (DE). Although disparities between the lowest and highest education group are smaller among men than among women, they have increased over time at a much higher rate as shown (see Appendix Table S4).

Finally, examination of chrDFLE 65 (see Table 3) show similar patterns as those observed for DFLE $_{65}$. Among women, there is a significant increase in the difference in chrDFLE 65 between the two higher education groups. Although among males these same differences contracted, differences in chrDFLE 65 between those with low education and those with high education expanded by more than 0.5 years (see Table S5 and Table S6 in the supplementary material).

\section{Discussion}

Recent studies suggest that despite continued gains in life expectancy, older adults in Spain spend more time with disability when compared with older generations [7, 8]. In this paper we use alternative data sources and two different indicators to confirm this unexpected expansion of disability and morbidity. Moreover, we augment findings to include an assessment of education differentials in levels and trends of both indicators.

The compression of morbidity observed in Spain in the 1980 and 90s [6] when mortality was decreasing sharply, may have been possible due to a contemporaneous reduction in age-specific

\footnotetext{
${ }^{5} \Delta D E$ refers to the difference in DFLE due to changes in disability fractions
} 
incidence of morbidity at younger ages. This is so because the incidence of the most dominant condition, e.g. CVD, remained unchanged or declined during the 80's and no new chronic illness gained a significant foothold. Our findings suggest that more recently these patterns have been overturned, as both DFLE $_{65}$ and chrDFLE 65 reveal expansion of both morbidity and disability among men and expansion of disability among women.

An equally important finding is that the observed declining trends in healthy life expectancy are mostly sustained by reductions among those with the lowest level of education, the groups that experienced the sharpest drop in DFLE $_{65}$. These unequal declines drive increases in disparities between educational groups, a phenomenon in line with recent findings showing similar increases in mortality disparities by education [21]. Similarly, the chrDFLE indicator also declines for men, and it does so across all groups although the decline is sharper among those with the lowest levels of education.

How can we explain these patterns?

The first explanation is simply that the observed trends are a result of artifacts that increase observed levels of prevalence with disability and chronic illnesses. Over the last decade or so, Spain has experienced rapid economic development, modernization, education expansion and, importantly, a massive increase of the reach and influence of the health sector. The combination of these changes could lead to improvements in screening and detection which may, in turn, inflate measures of chronic illnesses prevalence [30]. While plausible, this explanation fails to account for observed increases in education disparities, which should have diminished, not increased.

The second explanation is that observed increase in the risks of chronic illness and disability could have been fueled by a late epidemiological transition driven by behaviors associated with diet, sedentary life styles, jointly with increased consumption of tobacco and alcohol. The transition may have started well before the period under study, but its effects could only be observed with a generous time lag. If this is the case, it may well be that the full effects of the transition are not yet exhausted. This explanation is consistent with increases in educational disparities, for it is likely that it is among the lowest educated groups where the largest shifts in life styles have taken 
place [31]. Indeed, in Spain as elsewhere, the unequal adoption of new life styles that augments exposures to chronic illnesses have increased intra-group homogeneity and inter-group heterogeneity and thus may have generated new grounds for the emergence of health inequalities that are now reflected in our DFLE index.

Similar to the trends in social class patterns, also the observed gender differences in the chrDFLE indicator could stem from such time lagged effect. The stark gender differences in the chrDFLE indicator possibly reflect preexisting social barriers and/or gender-specific differences in the adaptation of risky behaviors such as smoking. Indeed, while smoker rates among men and women have converged over the past decades in Spain, among the cohorts born before 1960 the share of women who smoke or had smoked was marginal while up to $70 \%$ of all men in the cohort 1950-59 were either regular or former smoker [32, 33].

The third explanation is not part of a list of usual suspects. This is that, perhaps, the expansion of morbidity and disability is a cohort- based phenomenon. In particular, cohorts born between 1933 and 1943 may include a larger fraction of individuals who experienced adverse early conditions, namely, exposure to devastation brought about by the Spanish Civil War and its aftermath [3, 34, 35]. These exposures may have contributed to an increase in the cohorts' adult risks of metabolic and cardiovascular diseases [36, 37]. If this third explanation is correct, the decline in DFLE we observe during the period under examination is a transient phenomenon.

\subsection{Strenghts and Limitations}

This paper uses new data sources to confirm a recently detected expansion of morbidity in Spain, tried to trace down the origin of these trends and extended the existing empirical findings with an examination of education-specific trends and contributions.

The paper has a few shortcomings. First, like all studies of this type, estimates of DFLE and ChrDFLE are conditional on the definition of disability and chronic diseases. By including limitations in basic activity of daily living only, our DFLE indicator detects the occurrence of severe disability without offering any room to assess variation in severity. Thus, we are unable to test 
conjectures associated with dynamic equilibrium. By the same token, the indicator chrDFLE rests on reports about diagnosis of illnesses but does not capture variability in adherence and success of treatment and, hence, it is blind to severity as well.

Second, information on mortality during the period of observation is retrieved from matching individual records of the EDAD survey and vital statistics. As most data of this kind, ours is vulnerable to errors generated by imperfect linkages, including records that could not be linked at all.

Third, our estimates of chrDFLE from the EDAD questionnaire are indirect as they are based on prevalence of chronic illnesses among those with at least one limitation (see Appendix). Although the assumptions invoked to generate these indirect estimates are likely to be harmless, it would have been better to have avoided them altogether.

Finally, to our knowledge, the chrDFLE indicator has not been used widely or at all and, therefore, we cannot rely on past research to support its validity. In particular, we are not able to assess if and to what and extent observed changes in chrDFLE could be the result of improvements (deterioration) of screening and diagnosis.

\subsection{Concluding remarks}

Like recent studies in other countries [16, 38], we find evidence of an expansion of morbidity in Spain between 2008 and 2017. The bulk of the decline is associated with increases in time spent with disability and chronic disease and less so with changes in mortality. This pattern is more

pronounced among women as they experience smaller relative increases in life expectancy but higher prevalence of disability and illness. Significantly, we retrieve empirical evidence of salient disparities in DFLE and chrDFLE levels and trends across education groups. This is consistent with recent findings in other populations [24, 39, 40]. Not only are the size of these disparities relatively large in both periods but, more importantly, they increase over time. Future analyses enriched with measures of severity may shed more light on this and determine whether or not the increases in prevalence of chronic illness that explains the decline in chrDFLE is a reflection of 
improved screening and less a result of increased incidence.

\section{Conflict of Interest}

None declared.

\section{Code}

The code used to reproduce this analysis can be found in the following repository https:// gitlab.com/csic-echo/compression-morbidity. 


\section{Data Availability Statement}

A minimal sample of anonymized, individual level information, methodology and all questionnaires of the Spanish national survey on disability, autonomy, personal situations and dependency ("Encuesta sobre discapacidades, autonomía personal y situaciones de dependencia"; short: EDAD) and the Spanish National Health Survey 2017 ("Encuesta Nacional de Salud") can be downloaded from the website of the Spanish National Institute of Statistics (INE) without further costs. Link: https://www.ine.es/dyngs/INEbase/es/operacion.htm?c=Estadistica_C\& cid=1254736176782\&menu=resultados\&secc=1254736194716\&idp=1254735573175\#! tabs -

\section{3}

This data was used to to extract age-specific disability and chronic disease rates. The mortality follow-up information, used for the construction of education-specific life tables, cannot be shared publicly. The authors agreed upon this condition when signing the petition BE014/2018 (02.02.2018, attached in Spanish) with the INE. The INE department for sociodemographic statistics imposed these data restrictions to avoid the potential identification of survey participants, as the mortality data includes precise geographic and age-specific information. However, the data is available upon request from INE to all researcher who meet the criteria for access to confidential data.

For information on how to request and access this data, please consult the user service in person under the following address: National Statistics Institute. Área de atención a usuarios Paseo de la Castellana 183 (entrada por Estébanez Calderón 2) 28046 Madrid, Spain The other options to get in contact about data access with INE are to call the information hotline (+34 915839100$)$ or use the online consultation form: wWw. ine.es/infoine. 


\section{References}

1. Klenk, J., Keil, U., Jaensch, A., Christiansen, M. C. \& Nagel, G. Changes in Life Expectancy 1950-2010: Contributions from Age- and Disease-Specific Mortality in Selected Countries. Population Health Metrics 14, 20 (23, 2016).

2. Robine, J.-M. in International Handbook of Adult Mortality (eds Rogers, R. G. \& Crimmins, E. M.) 207-226 (Springer Netherlands, Dordrecht, 2011).

3. Gómez-Redondo, R. \& Boe, C. Decomposition Analysis of Spanish Life Expectancy at Birth: Evolution and Changes in the Components by Sex and Age. Demographic Research S4, 521$546(17,2005)$.

4. Mäki, N. et al. Educational Differences in Disability-Free Life Expectancy: A Comparative Study of Long-Standing Activity Limitation in Eight European Countries. Social Science \& Medicine 94, 1-8 (1, 2013).

5. Majer, I. M., Nusselder, W. J., Mackenbach, J. P. \& Kunst, A. E. Socioeconomic Inequalities in Life and Health Expectancies around Official Retirement Age in 10 Western-European Countries. J Epidemiol Community Health 65, 972-979 (1, 2011).

6. Sagardui-Villamor, J., Guallar-Castillón, P., García-Ferruelo, M., Banegas, J. R. \& RodríguezArtalejo, F. Trends in Disability and Disability-Free Life Expectancy Among Elderly People in Spain: 1986-1999. The Journals of Gerontology: Series A 60, 1028-1034 (1, 2005).

7. Walter, S. et al. No Evidence of Morbidity Compression in Spain: A Time Series Study Based on National Hospitalization Records. International Journal of Public Health 61, 729738 (2016).

8. Solé-Auró, A. \& Alcañiz, M. Are We Living Longer but Less Healthy? Trends in Mortality and Morbidity in Catalonia (Spain), 1994-2011. European Journal of Ageing 12, 61-70 (1, 2015).

9. Gruenberg, E. M. The Failures of Success. The Milbank Memorial Fund Quarterly. Health and Society 55, 3-24 (1977).

10. Fries, J. F. Aging, Natural Death, and the Compression of Morbidity. New England Journal of Medicine 303, 130-135 (1980).

11. Manton, K. G. Epidemiological, Demographic, and Social Correlates of Disability among the Elderly. The Milbank Quarterly 67, 13-58 (1989).

12. Hossin, M. Z., Östergren, O. \& Fors, S. Is the Association Between Late Life Morbidity and Disability Attenuated Over Time? Exploring the Dynamic Equilibrium of Morbidity Hypothesis. The Journals of Gerontology: Series B 74, e97-e106 (4, 2019).

13. Jeune, B. \& Brønnum-Hansen, H. Trends in Health Expectancy at Age 65 for Various Health Indicators, 1987-2005, Denmark. European Journal of Ageing 5, 279 (28, 2008). 
14. Cai, L. \& Lubitz, J. Was There Compression of Disability for Older Americans from 1992 to 2003? Demography 44, 479-495 (2007).

15. Crimmins, E. M., Zhang, Y. S., Kim, J. K. \& Levine, M. E. Changing Disease Prevalence, Incidence, and Mortality Among Older Cohorts: The Health and Retirement Study. The Journals of Gerontology: Series A 74, S21-S26 (Supplement_1 13, 2019).

16. Cambois, E., Blachier, A. \& Robine, J.-M. Aging and Health in France: An Unexpected Expansion of Disability in Mid-Adulthood over Recent Years. The European Journal of Public Health 23, 575-581 (1, 2013).

17. Hernández-Hernández, J. R., Heras-Gómez, F., Cordovilla-Pérez, M. R., Antolín-García, T. \& de Miguel, E. B. Incidence of Bronchopulmonary Carcinoma in Castile-Leon and Cantabria in 2007. Study from the Castile-Leon and Cantabria Respiratory Disease Society (SOCALPAR). Archivos de Bronconeumología ((English Edition)) 46, 7-14 (1, 2010).

18. De Pedro-Cuesta, J. et al. Prevalence of Dementia and Major Dementia Subtypes in Spanish Populations: A Reanalysis of Dementia Prevalence Surveys, 1990-2008. BMC Neurology 9, $55(19,2009)$.

19. Ruiz-Ramos, M., Escolar-Pujolar, A., Mayoral-Sánchez, E., Corral-San Laureano, F. \& FernándezFernández, I. La diabetes mellitus en España: mortalidad, prevalencia, incidencia, costes económicos y desigualdades. Gaceta Sanitaria 20, 15-24 (1, 2006).

20. Reques, L., Giráldez-García, C., Miqueleiz, E., Belza, M. J. \& Regidor, E. Educational Differences in Mortality and the Relative Importance of Different Causes of Death: A 7Year Follow-up Study of Spanish Adults. J Epidemiol Community Health 68, 1151-1160 (1, 2014).

21. Permanyer, I., Spijker, J., Blanes, A. \& Renteria, E. Longevity and Lifespan Variation by Educational Attainment in Spain: 1960-2015. Demography 55, 2045-2070 (1, 2018).

22. Solé-Auró, A., Martín, U. \& Domínguez Rodríguez, A. Educational Inequalities in Life and Healthy Life Expectancies among the 50-Plus in Spain. International Journal of Environmental Research and Public Health 17, 3558 (10 2020).

23. Solé-Auró, A., Beltrán-Sánchez, H. \& Crimmins, E. M. Are Differences in Disability-Free Life Expectancy by Gender, Race, and Education Widening at Older Ages? Population Research and Policy Review 34, 1-18 (1, 2015).

24. Matthews, R. J., Jagger, C. \& Hancock, R. M. Does Socio-Economic Advantage Lead to a Longer, Healthier Old Age? Social Science \& Medicine 62, 2489-2499 (1, 2006).

25. Cambois, E., Robine, J.-M. \& Hayward, M. D. Social Inequalities in Disability-Free Life Expectancy in the French Male Population, 1980-1991. Demography 38, 513-524 (1, 2001). 
26. Sullivan, D. F. A Single Index of Mortality and Morbidity. HSMHA Health Reports 86, 347354 (1971).

27. Arriaga, E. E. Measuring and Explaining the Change in Life Expectancies. Demography 21, 83-96 (1, 1984).

28. Nusselder, W. J. \& Looman, C. W. N. Decomposition of Differences in Health Expectancy by Cause. Demography 41, 315-334 (1, 2004).

29. Freedman, V. A., Schoeni, R. F., Martin, L. G. \& Cornman, J. C. Chronic Conditions and the Decline in Late-Life Disability. Demography 44, 459-477 (1, 2007).

30. Haase, C. B., Gyuricza, J. V. \& Brodersen, J. New Hypertension Guidance Risks Overdiagnosis and Overtreatment. BMJ 365 (12, 2019).

31. Palloni, A., Milesi, C., White, R. G. \& Turner, A. Early Childhood Health, Reproduction of Economic Inequalities and the Persistence of Health and Mortality Differentials. Social Science \& Medicine 68, 1574-1582 (1, 2009).

32. Schiaffino, A. et al. Gender and Educational Differences in Smoking Initiation Rates in Spain from 1948 to 1992. European Journal of Public Health 13, 56-60 (1, 2003).

33. Fernandez, E. et al. Prevalence of Cigarette Smoking by Birth Cohort among Males and Females in Spain, 1910-1990. European Journal of Cancer Prevention 12, 57-62 (2003).

34. Christiansen, T. The Reason Why: The Post Civil-War Agrarian Crisis in Spain (Universidad de Zaragoza, 2012).

35. Harrison, J. An Economic History of Modern Spain (Manchester University Press, 1978).

36. Barker, D. J. P. The Developmental Origins of Adult Disease. Journal of the American College of Nutrition 23, 588S-595S (sup6 1, 2004).

37. Ramirez, D. \& Haas, S. A. The Long-Arm of Conflict: How Timing Shapes the Impact of Childhood Exposure to War. Demography forthcoming (2020).

38. Kingston, A. et al. Is Late-Life Dependency Increasing or Not? A Comparison of the Cognitive Function and Ageing Studies (CFAS). The Lancet 390, 1676-1684 (7, 2017).

39. Cambois, E. et al. Educational Differentials in Disability Vary across and within Welfare Regimes: A Comparison of 26 European Countries in 2009. J Epidemiol Community Health 70, 331-338 (1, 2016).

40. Fouweather, T. et al. Comparison of Socio-Economic Indicators Explaining Inequalities in Healthy Life Years at Age 50 in Europe: 2005 and 2010. European Journal of Public Health 25, 978-983 (1, 2015). 


\section{Figures}

Figure 1: Observed Fraction with Functional Limitation (2008, 2017)

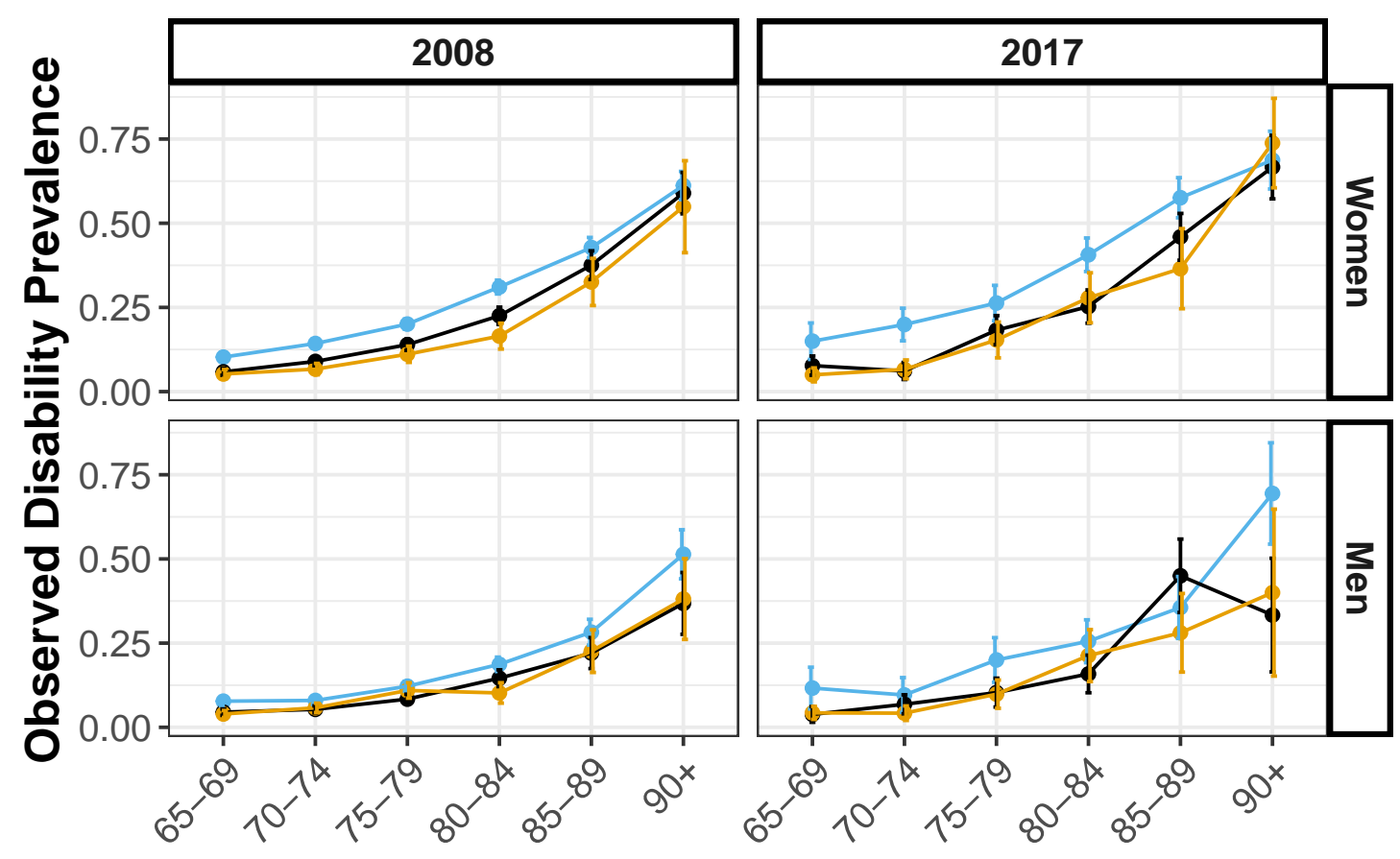

$\rightarrow$ low education $\rightarrow$ primary education $\bullet$ high education 


\section{Tables}

Table 1: DFLE and chrDFLE at Age 65 with $95 \%$ by Education and over Time

\begin{tabular}{|c|c|c|c|}
\hline & Low Education & Primary Education & High Education \\
\hline & \multicolumn{3}{|c|}{ Women 2008-2012 } \\
\hline \multirow[t]{2}{*}{$\begin{array}{l}\text { DFLE }_{65} \\
95 \% \mathrm{CIs} \\
\text { chrDFLE }_{65} \\
95 \% \text { CIs }\end{array}$} & $\begin{array}{c}16.041 \\
(15.757,16.361) \\
10.275 \\
(10.166,10.563)\end{array}$ & $\begin{array}{c}18.314 \\
(17.918,18.722) \\
11.747 \\
(11.613,12.236) \\
\end{array}$ & $\begin{array}{c}19.165 \\
(18.591,20.445) \\
12.574 \\
(12.145,13.581)\end{array}$ \\
\hline & \multicolumn{3}{|c|}{ Women 2013-2017 } \\
\hline \multirow[t]{2}{*}{$\begin{array}{l}\text { DFLE }_{65} \\
95 \% \text { CIs } \\
\text { chrDFLE } \\
95 \% \text { CIs } \\
95\end{array}$} & $\begin{array}{c}14.496 \\
(13.937,15.053) \\
11.234 \\
(10.608,11.859) \\
\end{array}$ & $\begin{array}{c}17.676 \\
(17.179,18.172) \\
13.342 \\
(12.710,13.948) \\
\end{array}$ & $\begin{array}{c}17.775 \\
(16.974,18.543) \\
15.450 \\
(14.538,16.355)\end{array}$ \\
\hline & \multicolumn{3}{|c|}{ Men 2008-2012 } \\
\hline $\begin{array}{l}\text { DFLE }_{65} \\
95 \% \text { CIs } \\
\text { chrDFLE } 65 \\
95 \% \text { CIs }\end{array}$ & $\begin{array}{c}15.166 \\
(14.800,15.472) \\
9.594 \\
(9.474,9.920)\end{array}$ & $\begin{array}{c}17.045 \\
(16.574,17.513) \\
10.780 \\
(10.537,11.117) \\
\text { Men 2013-2017 }\end{array}$ & $\begin{array}{c}16.958 \\
(16.421,17.690) \\
10.883 \\
(10.443,11.315)\end{array}$ \\
\hline $\begin{array}{l}\text { DFLE }_{65} \\
95 \% \text { CIs } \\
\text { chrDFLE } 65 \\
95 \% \text { CIs }\end{array}$ & $\begin{array}{c}13.839 \\
(13.211,14.413) \\
8.144 \\
(7.423,8.874)\end{array}$ & $\begin{array}{c}16.623 \\
(16.102,17.203) \\
9.964 \\
(9.342,10.694)\end{array}$ & $\begin{array}{c}16.792 \\
(16.113,17.559) \\
9.752 \\
(8.999,10.516)\end{array}$ \\
\hline
\end{tabular}


Table 2: Decomposition of Period Differences in DFLE 65 with 95\% CIs by Education

\begin{tabular}{l|c|c|c}
\hline & Low Education & Primary Education & High Education \\
\hline \multicolumn{3}{|c|}{ Women } & \\
\hline $95 \%$ CIs & $(-2.186,-0.916)$ & $(-1.353,0.060)$ & $(-2.914,-0.478)$ \\
\hline$\Delta \mathrm{ME}$ & 0.042 & 0.073 & -0.070 \\
$95 \% \mathrm{CIs}$ & $(-0.240,0.356)$ & $(-0.295,0.438)$ & $(-1.001,0.354)$ \\
\hline$\Delta \mathrm{DE}$ & -1.587 & -0.711 & -1.319 \\
$95 \% \mathrm{CIs}$ & $(-2.131,-1.047)$ & $(-1.324,-0.150)$ & $(-2.351,-0.421)$ \\
\hline & & Men & \\
\hline$\Delta \mathrm{DFLE} 65$ & -1.327 & -0.421 & -0.166 \\
$95 \% \mathrm{CIs}$ & $(-1.964,-0.610)$ & $(-1.117,0.342)$ & $(-1.176,0.774)$ \\
\hline$\Delta \mathrm{ME}$ & -0.256 & 0.0763 & 0.186 \\
$95 \% \mathrm{CIs}$ & $(-0.631,0.152)$ & $(-0.396,0.544)$ & $(-0.481,0.754)$ \\
\hline$\Delta \mathrm{DE}$ & -1.067 & -0.497 & -0.353 \\
$95 \% \mathrm{CIs}$ & $(-1.607,-0.478)$ & $(-1.002,0.040)$ & $(-1.083,0.347)$ \\
\hline
\end{tabular}

The notation ME refers to the impact of mortality on the DFLE given that disability rates remained constant. DE notes the effect of changes in disability rates on DFLE when mortality rates would remain constant over time. 
Table 3: Decomposition of Period Differences in ChrFLE $_{65}$ with $95 \%$ Cis by Education

\begin{tabular}{l|c|c|c}
\hline & Low Education & Primary Education & High Education \\
\hline & \multicolumn{3}{|c}{ Women } \\
\hline$\Delta$ chrDFLE $_{65}$ & 0.959 & 1.595 & 2.876 \\
$95 \%$ CIs & $(0.231,1.546)$ & $(0.702,2.100)$ & $(1.361,3.729)$ \\
\hline$\Delta \mathrm{ME}$ & 0.035 & 0.025 & 0.090 \\
$95 \%$ CIs & $(-0.198,0.290)$ & $(-0.289,0.315)$ & $(-1.003,0.531)$ \\
\hline$\Delta$ CHRE & 0.923 & 1.570 & 2.786 \\
$95 \%$ CIs & $(0.226,1.452)$ & $(0.767,2.015)$ & $(1.908,3.676)$ \\
\hline & & Men & -1.131 \\
\hline$\Delta$ chrDFLE 65 & -1.449 & -0.815 & $(-1.949,-0.280)$ \\
$95 \%$ CIs & $(-2.313,-0.801)$ & $(-1.550,-0.076)$ & 0.106 \\
\hline$\Delta$ ME & -0.168 & 0.049 & $(-0.337,0.485)$ \\
$95 \%$ CIs & $(-0.418,0.121)$ & $(-0.249,0.349)$ & -1.237 \\
\hline CHRE & -1.281 & -0.865 & $(-1.917,-0.408)$ \\
$95 \%$ CIs & $(-2.108,-0.706)$ & $(-1.521,-0.226)$ &
\end{tabular}

The notation ME refers to the impact of mortality on the chrDFLE given that chronic disease rates remained constant. DE notes the effect of changes in disability rates on chrDFLE when mortality rates would remain constant over time. 


\section{Appendix}

\section{Appendix 1 - Additional information about the linkage of the EDAD survey}

The INE department for socio-demographic statistics used a personal identifier, such as the Spanish national ID number (DNI/NIE), to link the 258,187 interviewees who participated in the National Survey on Disability, Personal Autonomy, and Dependency (EDAD, Spanish: Encuesta sobre Discapacidad, Autonomia personal y Situaciones de Dependencia) with a monthly updated Spanish population register (Spanish: Padrón) and the statistics for natural population movement (MNP, Spanish: Movimiento natural de la población). Padrón data was used to assess the timing of changes in household size. The MNP, which combines individual level information from various civil registers, contains more detailed information on the causes for the increase or reduction of household size. In the case of a reduction of household size the MNP data allowed for distinguishing if such a reduction was caused by the emigration or death of one of the household members.

The EDAD survey was not designed to be linked to register data. Especially in the EDAD, a substantial number of individuals could not be identified because their id information was missing. As INE informed us, 50,658 personal identifier were missing in the original data file and could not be linked to the population registers.

INE assumes that this information is missing at random. In addition, 1783 individuals with valid id information could not be unequivocally matched with individuals in the registers and were excluded from the analysis. Of these cases, 1744 individuals could not be linked to the MNP but "left" the population according to the Padrón. Many of these cases are presumably unregistered deaths, which might not yet have been recorded in the MNP at the time of data collection.

More information about the sampling and underlying methodology of the EDAD survey can be found on the INE website (information in Spanish; Link: Metodología EDAD The INE website further contains additional information about the Padrón (Information Padrón), the MNP (Information MNP), and the Spanish National Health Survey, which was used to obtain disability fractions and information on chronic diseases for the second observation period (Information ENSE 2017). 


\section{Appendix 2 - Indirect estimation of fraction with chronic diseases in 2008}

The EDAD survey elicits information on a variety of limitations, ranging from problems with vision to severe functional limitations. Interviewees who acknowledged problems with any one of 44 daily activities were asked follow-up questions on their health state and diagnosed chronic conditions. Therefore, the data did not allow us to estimate the fractions of those with chronic diseases when they had no disability. To circumvent this issue, we approximated age and sexspecific fractions of those with chronic disease but without disability based on two other data points for which nationally representative data was available by age, sex, and comparable education categories. The estimates are extracted from the Spanish National Health Survey of 2011 and 2017 (Encuesta Nacional de Salud and the comparable Spanish part of European Health Survey 2014 (Encuesta Europea de Salud. Under the assumption that the risk of moving from a non-disabled state with chronic condition to a state with disability and chronic condition remains constant over time, we estimated the fraction of those with chronic condition in 2008 as sum of those with chronic condition and disability and the newly estimated fraction of those without disability based on the three health surveys. 


\section{Appendix 3 - Chronic Disease Rates}

Figure S1: Observed Fraction of Women with Selected Chronic Diseases $(2008,2017)$
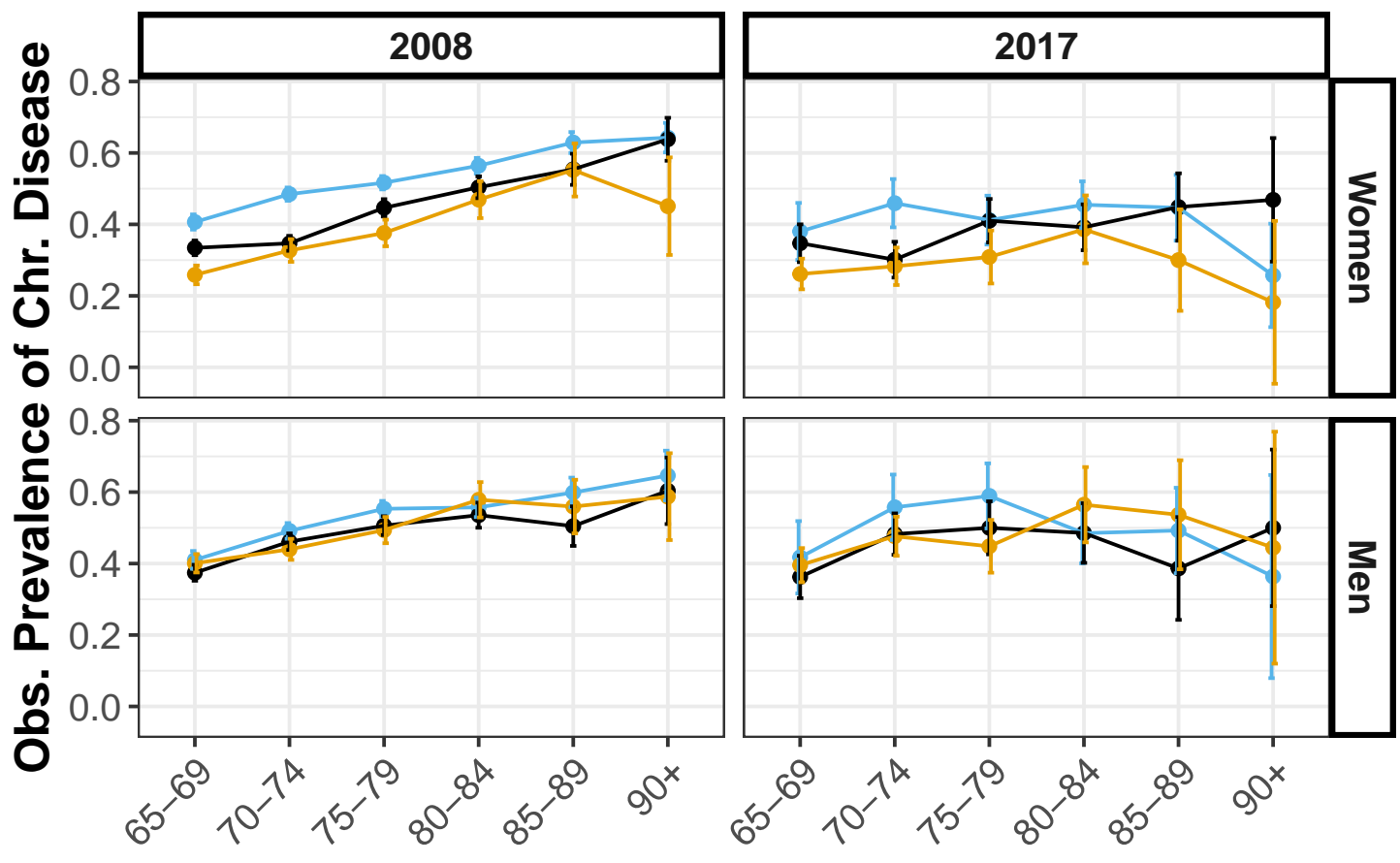

$\rightarrow$ low education $\rightarrow$ primary education $\rightarrow$ high education 


\section{Appendix 4 - Life Tables by Gender and Education}

Table S1: Life Expectancy by Gender, Education and Year with 95\% Confidence Intervals

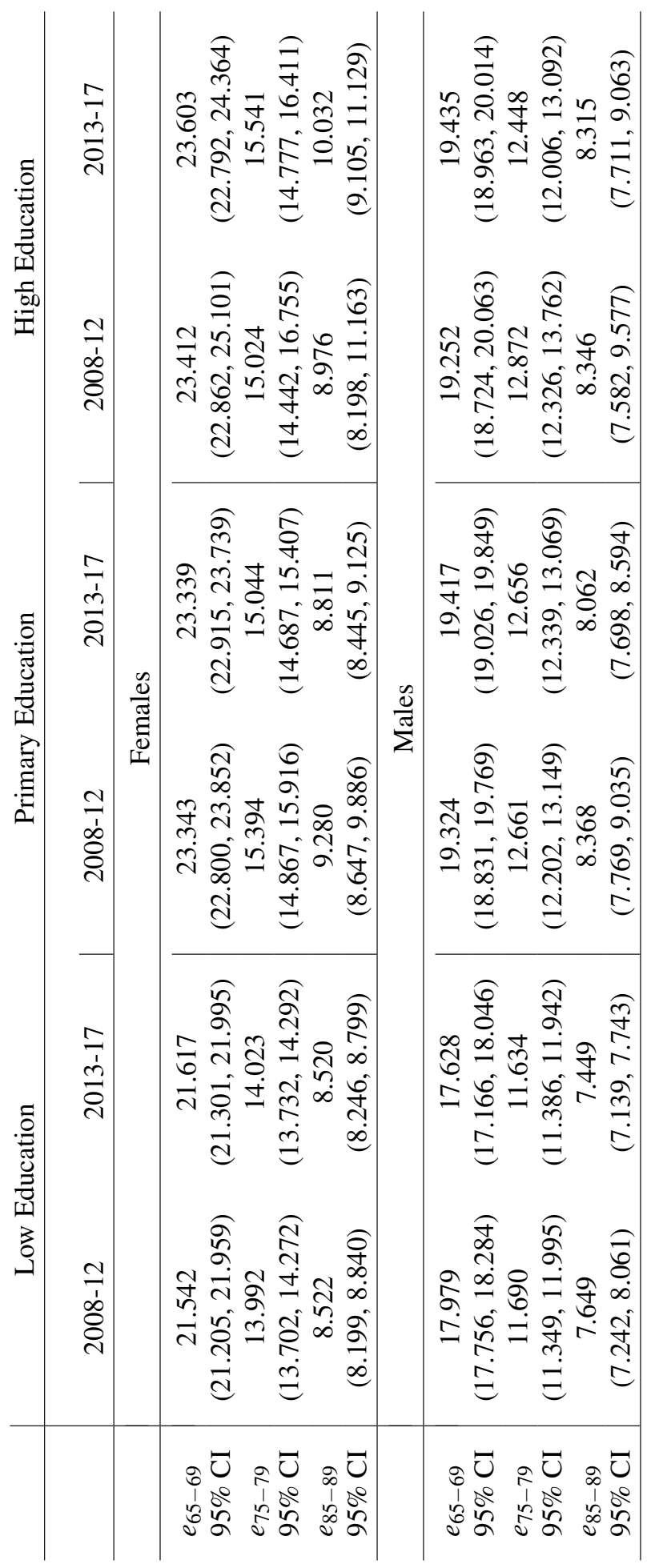




\section{Appendix 5 - Tables Decomposition - Comparison between Education Groups}

Table S2: Estimated Life Expectancy, DFLE and ChrFLE with 95\% CIs at Age 65 by Sex and Period

\begin{tabular}{l|c|c}
\hline & Women & Men \\
\hline & \multicolumn{2}{|c}{$2008-2012$} \\
\hline $\mathrm{ex}_{65}$ & 22.384 & 18.648 \\
$95 \%$ CIs & $(22.135,22.662)$ & $(18.382,18.901)$ \\
\hline DFLE $_{65}$ & 17.195 & 16.109 \\
$95 \%$ CIs & $(16.972,17.418)$ & $(15.860,16.359)$ \\
\hline chrDFLE $_{65}$ & 11.112 & 10.344 \\
$95 \%$ CIs & $(10.990,11.309)$ & $(10.121,10.443)$ \\
\hline & \multicolumn{2}{|c}{$2013-2017$} \\
\hline ex$_{65}$ & 22.515 & 18.784 \\
95\% CIs & $(22.268,22.761)$ & $(18.563,19.031)$ \\
\hline DFLE $_{65}$ & 16.455 & 15.751 \\
95\% CIs & $(16.150,16.759)$ & $(15.418,16.068)$ \\
\hline chrDFLE 65 & 13.111 & 9.421 \\
95\% CIs & $(12.753,13.508)$ & $(9.051,9.828)$ \\
\hline & \multicolumn{2}{|c}{}
\end{tabular}


Table S3: Decomposition of Education Group Differences in DFLE 65 with 95\% CIs by Period (Women)

\begin{tabular}{l|c|c}
\hline & Low Education vs. High Education & Primary Education vs. High Education \\
\hline & \multicolumn{2}{|c}{$2008-2012$} \\
\hline$\Delta$ DFLE $_{65}$ & 3.123 & 0.850 \\
$95 \%$ CIs & $(2.506,4.434)$ & $(0.177,2.104)$ \\
\hline$\Delta$ MOR & 1.214 & 0.109 \\
$95 \%$ CIs & $(0.838,2.098)$ & $(-0.261,1.047)$ \\
\hline$\Delta$ DE & 1.909 & 0.741 \\
$95 \%$ CIs & $(1.406,2.491)$ & $(0.166,1.391)$ \\
\hline & & $2013-2017$ \\
\hline DFLE 65 & 3.279 & 0.099 \\
$95 \%$ CIs & $(2.219,4.172)$ & $(-0.829,1.038)$ \\
\hline$\Delta$ MOR & 0.927 & -0.053 \\
$95 \%$ CIs & $(0.867,1.266)$ & $(-0.261,1.047)$ \\
\hline$\Delta$ DE & 2.352 & 0.152 \\
$95 \%$ CIs & $(1.467,3.138)$ & $(-0.667,0.983)$ \\
\hline
\end{tabular}

The notation ME refers to the impact of mortality on the DFLE given that disability rates remained constant. DE notes the effect of changes in disability rates on DFLE when mortality rates would remain constant over time. 
Table S4: Decomposition of Education Group Differences in DFLE 65 with 95\% CIs by Period (Men)

\begin{tabular}{l|c|c}
\hline & Low Education vs. High Education & Primary Education vs. High Education \\
\hline & \multicolumn{2}{|c}{$2008-2012$} \\
\hline$\Delta$ DFLE $_{65}$ & 1.792 & -0.087 \\
$95 \%$ CIs & $(1.167,2.069)$ & $(-0.738,0.773)$ \\
\hline$\Delta$ MOR & 0.920 & -0.085 \\
$95 \%$ CIs & $(0.482,1.589)$ & $(-0.597,0.612)$ \\
\hline$\Delta$ DE & 0.872 & -0.002 \\
$95 \%$ CIs & $(0.513,1.218)$ & $(-0.003,0.401)$ \\
\hline & & $2013-2017$ \\
\hline DFLE 65 & 2.953 & 0.168 \\
$95 \%$ CIs & $(2.069,3.893)$ & $(-0.711,1.091)$ \\
\hline$\Delta$ MOR & 1.277 & 0.025 \\
$95 \%$ CIs & $(0.842,1.812)$ & $(-0.461,0.531)$ \\
\hline$\Delta$ DE & 1.676 & 0.143 \\
$95 \%$ CIs & $(0.851,2.440)$ & $(-0.631,0.879)$ \\
\hline
\end{tabular}

The notation ME refers to the impact of mortality on the DFLE given that disability rates remained constant. DE notes the effect of changes in disability rates on DFLE when mortality rates would remain constant over time. 
Table S5: Decomposition of Education Group Differences in ChrFLE 65 with $95 \%$ CIs by Period (Women)

\begin{tabular}{l|c|c}
\hline & Low Education vs. High Education & Primary Education vs. High Education \\
\hline & \multicolumn{2}{|c}{$2008-2012$} \\
\hline ChrFE $_{65}$ & 2.312 & 0.773 \\
$95 \%$ CIs & $(1.756,5.307)$ & $(0.175,1.726)$ \\
\hline$\Delta$ ME & 0.849 & 0.050 \\
$95 \%$ CIs & $(0.579,1.577)$ & $(-0.248,0.847)$ \\
\hline$\Delta$ CHRE & 1.463 & 0.723 \\
$95 \%$ CIs & $(1.045,1.857)$ & $(0.220,1.104)$ \\
\hline & & $2013-2017$ \\
\hline ChrFLE & 4.216 & 2.108 \\
$95 \%$ CIs & $(3.119,5.307)$ & $(0.979,3.172)$ \\
\hline$\Delta$ ME & 1.140 & 0.082 \\
$95 \%$ CIs & $(0.603,1.631)$ & $(-0.335,0.567)$ \\
\hline$\Delta$ CHRE & 3.075 & 1.991 \\
$95 \%$ CIs & $(2.119,4.111)$ & $(0.946,3.019)$ \\
\hline
\end{tabular}

The notation ME refers to the impact of mortality on the chrDFLE given that disability rates remained constant. CHRE notes the effect of changes in chronic disease rates on chrDFLE when mortality rates would remain constant over time. 
Table S6: Decomposition of Education Group Differences in ChrFLE 65 with $95 \%$ CIs by Period (Men)

\begin{tabular}{|c|c|c|}
\hline & Low Education vs. High Education & Primary Education vs. High Education \\
\hline & \multicolumn{2}{|c|}{ 2008-2012 } \\
\hline $\begin{array}{l}\Delta \operatorname{chrDFLE}_{65} \\
95 \% \text { CIs }\end{array}$ & $\begin{array}{c}1.084 \\
(0.667,1.674)\end{array}$ & $\begin{array}{c}-0.075 \\
(-0.455,0.600)\end{array}$ \\
\hline $\begin{array}{l}\Delta \mathrm{ME} \\
95 \% \mathrm{CIs}\end{array}$ & $\begin{array}{c}0.660 \\
(0.338,1.118) \\
\end{array}$ & $\begin{array}{c}-0.044 \\
(-0.396,0.438) \\
\end{array}$ \\
\hline \multirow[t]{2}{*}{$\begin{array}{l}\Delta \mathrm{CHRE} \\
95 \% \mathrm{CIs}\end{array}$} & $\begin{array}{c}0.424 \\
(0.217,0.658) \\
\end{array}$ & $\begin{array}{c}0.012 \\
(-0.249,0.273)\end{array}$ \\
\hline & \multicolumn{2}{|c|}{$2013-2017$} \\
\hline $\begin{array}{l}\Delta \text { chrDFLE }_{65} \\
95 \% \text { CIs }\end{array}$ & $\begin{array}{c}1.607 \\
(0.536,2.654)\end{array}$ & $\begin{array}{c}-0.212 \\
(-1.274,0.759)\end{array}$ \\
\hline $\begin{array}{l}\Delta \mathrm{ME} \\
95 \% \mathrm{CIs}\end{array}$ & $\begin{array}{c}0.827 \\
(0.525,1.175) \\
\end{array}$ & $\begin{array}{c}0.012 \\
(-0.281,0.322) \\
\end{array}$ \\
\hline $\begin{array}{l}\Delta \mathrm{CHRE} \\
95 \% \mathrm{CIs}\end{array}$ & $\begin{array}{c}0.790 \\
(-0.209,1.756)\end{array}$ & $\begin{array}{c}-0.223 \\
(-1.289,0.759)\end{array}$ \\
\hline
\end{tabular}

The notation ME refers to the impact of mortality on the chrDFLE given that chronic disease rates remained constant. DE notes the effect of changes in disability rates on chrDFLE when mortality rates would remain constant over time. 